\title{
Blowing Steam into the Boundary Layer on the Outer Wall of the Axial Exhaust Hood
}

\author{
David Tupý ${ }^{1,2, *}$, and Václav Sláma ${ }^{1,2}$ \\ ${ }^{1}$ Doosan Skoda Power s.r.o., Experimental Research of Flow, Tylova 1/57, 301 28, Pilsen, \\ Czech Republic \\ ${ }^{2}$ University of West Bohemia, Power System Engineering Department, Univerzitní 8, 306 14, Pilsen, \\ Czech Republic
}

\begin{abstract}
The paper is devoted to experimental verification of flow on the steam turbine axial exhaust hood model. The main goal of the submitted paper is to evaluate the influence of additional air blowing into the boundary layer in the axial exhaust hood on the change of the pressure recovery coefficient. The experimental model was supplemented with a blowing track and fitted with a centric orifice for mass flow measurement. Several variants were measured with various types of inner struts and supports inside the hood for two values of Mach number at the diffuser inlet.
\end{abstract}

\section{Introduction}

The paper deals with a controlled blowing into the boundary layer in axial exhaust hoods. The axial exhaust hoods (diffusers) are at present an often used type of steam turbine outlet. These hoods are mainly used for turbines of lower outputs up to $180 \mathrm{MW}$, when the condenser is located at the same height level.

The main task of the hood is to take the steam from the steam turbine last stage to the condenser with minimal losses or even with a pressure gain and in this way to increase the last stage enthalpy drop. Reaching a higher enthalpy drop contributes to the increase of turbomachine efficiency. In order to maximize the efficiency, it is necessary to minimalize energy losses incurred at the outlet of the last stage blade cascade, i.e. at the place of the highest velocities. Energy losses are influenced mainly by the shape of the diffuser and the size and shape of the exhaust hood itself. The exhaust hood of condensing turbines operates at the pressure lower than the atmospheric pressure. From the strength and stiffness point of view the exhaust hood must be supplemented with inner struts and supports. A number of technological pipelines go through the hood area - such as oil pipes, or pipes with gland or pipes to steam vents. All the mentioned obstacles are associated with significant energy losses of the flowing steam leaving the last stage.

The steam leaving the flow part does not do any useful work, but its parameters can have a significant influence on the turbomachine efficiency and durability. In other words, by suitable adjustment of outlet flow parameters and by minimizing losses it is possible to achieve greater enthalpy drop in the turbine last stage. The shape of limiting walls, the shape,

\footnotetext{
*Corresponding author: david.tupy@doosan.com
} 
number and location of inner struts and other elements has a fundamental influence on the aerodynamic efficiency of the exhaust hood. Efforts are also made to equalize the steam flow entering the condenser and thereby to improve the vibration characteristics of the bypassed pipe bundles.

Lowering energy losses and conversion of outlet kinetic energy to potential energy results in extension of the expansion line (increase in enthalpy drop) between the inlet and outlet of the last stage. This increase in enthalpy drop contributes to increase in the last stage efficiency and thus the efficiency of the whole turbomachine. This effect usually occurs at subsonic velocities at the diffuser inlet (about $0.4-0.7 \mathrm{Ma}$ ), whose cross section increases towards the outlet. At limit operating conditions for maximal flow rate and very low back pressure, velocities may approach speed of sound and the state of the so called aerodynamic blockage. However, this state does not correspond with common operating conditions, thus extreme conditions won't be considered in this paper. When designing an exhaust hood general requirements must be considered related with achieving adequate efficiency and reliability of the machine. The construction design of the exhaust hood should guarantee the following: separation of the working medium from the steam turbine last stage in the required direction with minimal aerodynamic losses, uniform pressure field behind the last stage of the turbine, uniform velocity field at the outlet of the nozzle, stationary and stable flow of the working medium, high rigidity of the outlet nozzle and acceptable overall dimensions of the construction.

The construction design of the last stage of the blade machine has a fundamental influence on fulfilling the above mentioned requirements. Flow in the exhaust hood depends on the outlet flow field from the last blade stage of the turbine. Among the parameters that basically influence the flow field behaviour is the axial outlet velocity, meridial and circumferential flow angle, the entire pressure profile along the blade, intensity of the turbulence etc. These parameters change with the operating condition of the machine that may not always work in the design regime. With different loads mainly velocity components and total pressure profile change. Last but not least the flow is influenced by the shape of the hood and its inner arrangement and inner struts. Increased attention must be paid to this complex issue. The exhaust hood design is obviously a complicated process during which many compromises must be made.

The paper deals with measurement on a reduced model of the exhaust hood with blowing into the boundary layer. The main aim of the work is to define the influence of blowing on the pressure recovery coefficient. In the paper, the description of the experimental device and measurements on the exhaust hood will be presented followed by the description of methodology used and the evaluation of the obtained results.

\section{Possibilities to reduce energy losses}

For most operating modes the exhaust hood must be designed as an expanding channel, in other words, as a diffuser. As the working medium flows through the exhaust hood (diffuser) as mentioned above, energy losses occur. Total losses in the exhaust hood consist of losses in the exhaust hood itself and outlet velocity losses. Losses in the exhaust hood are formed by friction losses and local losses (obstacles in the flow, sudden diffusion, flow separation from the walls etc.). In order to lower energy losses it is necessary to lower one or several of these partial losses. Loss energy of the exhaust hood is always determined using total losses coefficient $\zeta_{C}$.

There are several ways to reduce losses occurred in flow with flow separation from the diffuser wall. These are methods that try to remove or shift the separation further along the flow. Behind the flow separation points vortices and energy dissipation occur, which leads to increase in energy losses in the diffuser. 
The flowing medium and the exhaust hood construction fundamentally influence friction losses and related outlet velocity losses. It is possible to reduce outlet flow velocity by adding meshes or cascades at the exhaust hood, but it leads to loss increase by friction. It is important to assess individual aspects and then to choose which loss will prevail.

Among the methods used to lower energy loss are for example: sucking boundary layer, tangential blowing into the boundary layer, transversal blowing into the boundary layer, introducing the synthetized jet, cutting the boundary layer and others. The description of individual methods is described e.g. in reference [1].

One of the ways to influence actively the flow in the exhaust hood is tangential blowing of steam taken from the hollow rotor blades back to the boundary layer on the inner wall of the exhaust hood. By increasing the boundary layer energy it is possible to achieve a shift in the flow separation point from the by-passed wall or to avoid the boundary layer separation completely. However, it is always the question of choosing the right blown amount. Using many numerical simulations it was found out that the positive influence of a precisely dimensioned blowing corresponds to an improvement of pressure recovery coefficient $c_{p}$ by $2 \%$, which is a minimal contribution. In order to verify the influence of the blown amount, measurement was carried out on an experimental model.

A similar experiment was studied, inter alia, by Volkmer, Hirschmann, Casey and Montgomery [2], who examined how the flow velocity through radial clearance of stator blades $c_{\text {tip }}$ influences the pressure recovery coefficient $c_{p}$. The experiment was carried out on the general axial exhaust hood of a gas turbine. The flow through radial clearance was replaced by blowing additional flow to the annular part of the diffuser. It is precisely because of this fact that both experiments are very similar in nature. Based on the studies, the influence of flow blowing on the pressure recovery coefficient can be divided into three categories. For low velocities of the blown flow first the pressure recovery coefficient drops, and the minimal coefficient value is reached at the velocity ratio $c_{\text {tip }} / c_{\text {main }}=0.25$. The second area is in the range $c_{\text {tip }} / c_{\text {main }} \in(0.25 ; 1.2)$. A significant increase of the pressure recovery coefficient occurs when the velocity ratio ranges from 1 to 1.2 . In this area the boundary layer does not separate from the diffuser wall. The third area is reached when the additional flow reaches high velocities. The velocity ratio is 1.6 and higher. In this case the boundary layer does not separate from the diffuser wall either; however, the flow separation on the inner charge is significantly influenced, which is demonstrated by a considerable increase in total pressure losses. The dependence of ratio $c_{p, S 5} / c_{p, V R 0, S 5}$ on ratio $c_{\text {tip }} / c_{\text {main }}$ in plane $S 5$ for two different velocities of the blown flow is shown in Figure 1, where:

S5 - plane near the axial exhaust hood outlet,

$\begin{array}{ll}c_{p, S 5} & \text { - pressure coefficient in plane S5 during flow blowing, } \\ c_{p, V R 0, S 5} & \text { - pressure coefficient in plane S5 during zero velocity ratio (without flow } \\ & \text { blowing), } \\ c_{\text {tip }} & \text { - velocity of blown additional flow, } \\ c_{\text {main }} & \text { - velocity of the main flow. }\end{array}$ 


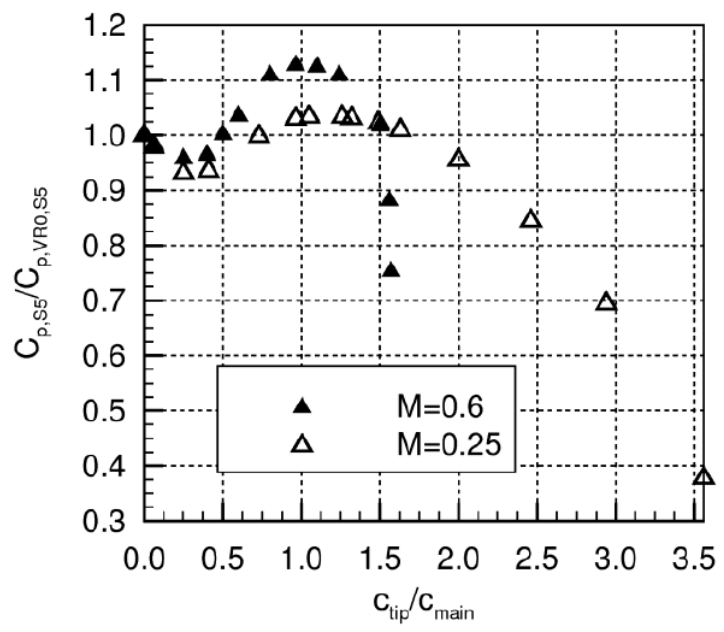

Fig. 1. Dependence ratio $c_{p, S 5} / c_{p, V R 0, S 5}$ on ratio $c_{t i p} / c_{\text {main }}[2]$.

\section{Experimental device}

Blowing into boundary layer on the outer wall of the axial exhaust hood was implemented on an experimental device located in the laboratory of the company Doosan Śkoda Power s.r.o. The device corresponds with the reduced model of the axial exhaust hood of the modern design steam turbine with the output of $35 \mathrm{MW}$ supplemented with a circular extension pipe. The model is attached to the aerodynamic wind tunnel outlet, see Figure $2 \mathrm{a}$ ). The detailed description of the wind tunnel is given e.g. in [3].

Using previous measurements on the model, optimal geometry was determined of the exhaust hood and configuration of the inner struts and supports. Among the observed parameters was mainly the velocity profile at the exhaust hood inlet, profile of the circumferential angle $\alpha_{1}$ at the hood inlet and geometrical configuration of inner struts. Altogether three types of stationary blade cascades were tested (Var. 6, 7 and 8), whose task was to provide the required circumferential angle at the exhaust hood inlet. The circumferential angle profile along the blade height for all three blade cascades can be seen in Fig. 2 b). Two sets of inner struts were also tested. These were circular and flat struts. Measurements were done for two values of Mach number at the diffuser inlet, namely 0.2 and $0.4 \mathrm{Ma}$. Given the limitations of the wind tunnel it was not possible to reach higher levels of Mach number. A suitable configuration was chosen regarding the highest level of pressure recovery coefficient $c_{p}$. The description of obtained results is given more in detail in e.g. [3], [4], [5]. Based on obtained results the most suitable variant seems to be Var. 6. For this reason the blowing was only done on this variant of the stationary cascade. 


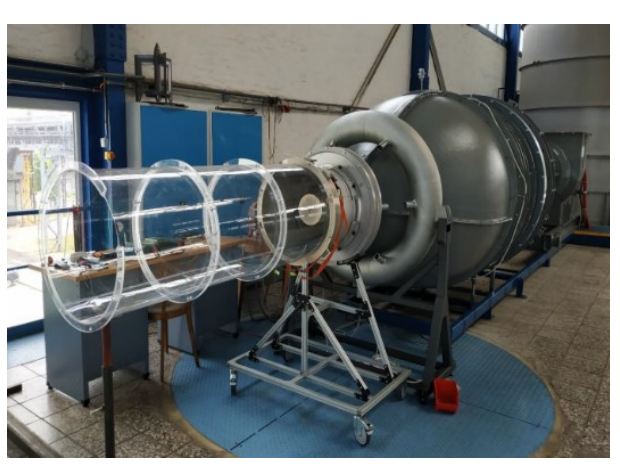

a)

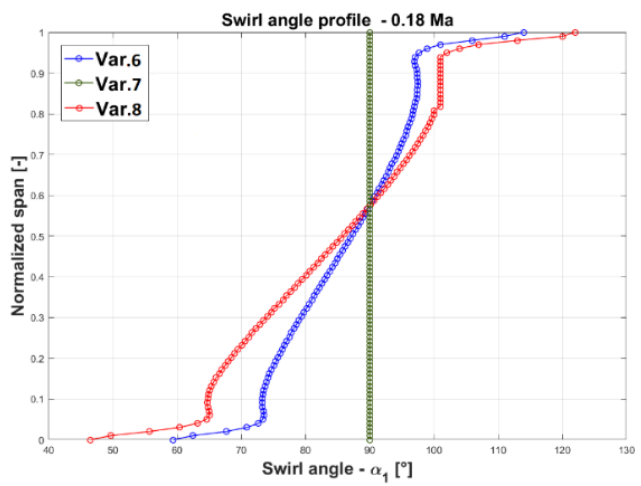

b)

Fig. 2. a) Exhaust hood model; b) Profile of circumferential angle $\alpha_{1}$.

\section{Measurement description}

The exhaust hood is consisted from an annular and conical part. In practice the condenser can be placed at a long distance (even several tens of meters) from the steam turbine. For this reason a circular extension pipe is attached to the conical part of the diffuser. In Fig. 3 a simplified scheme is shown with marked important planes:

- plane 0 - plane before the blade cascade,

- plane 1 - exhaust hood inlet,

- $\quad$ plane 2 - exhaust hood outlet,

- $\quad$ plane 3-circular extension pipe outlet to the open space.

A part of the exhaust hood is a blowing chamber that enables connection of an air pressure source and its controlled blowing using tangential slot into the boundary layer on the outer wall of the hood, which can have a significant influence on the final loss or pressure coefficient of the diffuser. The mass flow of the air blown into the circumferential slot is measured using a centric orifice. Behind the centric orifice a limiting orifice is placed that can, for a steady flow rate, lower the pressure of liquid at the orifice outlet to the required level, in other words it serves as a hydraulic resistance. The width of the blowing slot is $5 \mathrm{~mm}$ and cannot be changed. In the picture it is possible to observe the blowing track and corresponding pressure measurements before and behind the orifice that serve for determining mass flow. To determine the mass flow, it is also necessary to determine the density of the flowing air. In order to calculate the density it is necessary to measure the air temperature located in the distance specified by standard ČSN EN ISO 5167-2 [6].

Pressures on the exhaust hood model were measured using Prandtl probe, multi-hole pneumatic probes and static pressure taps. For pressure measurements three intelligent pressure transducers NetScanner 9116 were used with the range of 15 psi, 1 psi and 10 inches of water column. The barometric pressure was measured using pressure transducer Rosemount $3051 \mathrm{~S}$ ultra with measuring range $0 \div 207 \mathrm{kPa}$. For pressure measurement on the orifice two pressure transducers from the company Rosemount with the range of $700 \mathrm{kPa}$ and $5 \mathrm{kPa}$ with the usage of $\mathrm{A} / \mathrm{D}$ convertor NI 9203. The temperature of the blown air was measured using thermocouple of type K using convertor NI 9213. The remaining two temperatures were taken using resistance thermometer Pt100 using A/D convertor NI 9217. Convertors were set in chassis NI cDAQ 9178. 


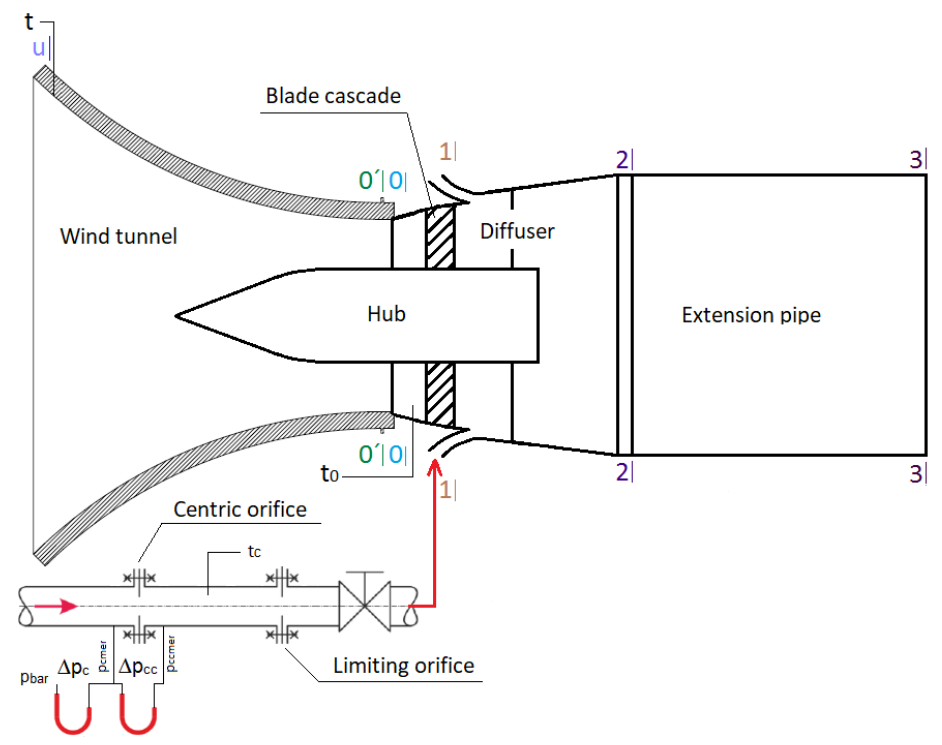

Fig. 3. Measurement scheme.

The main task of the work was to find out the influence of blowing into boundary layer on the pressure recovery coefficient $c_{p}$. Based on the knowledge of characteristic parameters of the blade cascade (the ratio of dynamic pressure before and behind the blade cascade $p_{p}$; loss coefficient of the cascade $\zeta_{m}$ ) it was possible to carry out measurements in the so called simple mode, when the Prandtl probe is placed in one reference position before the blade cascade (in plane 0). In the equations mentioned below there are corresponding total pressures $-p_{c}$, static pressures $-p_{s}$ a dynamic pressures $-p_{d}$. Using characteristic parameters it is possible to determine the loss coefficient of the diffuser $\zeta_{D}$ from the following relation:

$$
\zeta_{D}=\left(\frac{\Delta p_{c 0}-\Delta p_{s 2}}{p_{d 0}}-\zeta_{m}\right) \cdot \frac{p_{d 0}}{p_{d 1}}==\left(\zeta_{m D}-\zeta_{m}\right) \cdot p_{p}
$$

Pressure recovery coefficient $c_{p D}$ can be then determined as follows:

$$
c_{p D}=1-\zeta_{D}
$$

The loss coefficient of the diffuser supplemented with the already mentioned circular extension pipe $\zeta_{C}$ can be calculated using the relation:

$$
\zeta_{C}=\left(\frac{\Delta p_{c 0}}{p_{d 0}}-\zeta_{m}\right) \cdot \frac{p_{d 0}}{p_{d 1}}=\left(\zeta_{m C}-\zeta_{m}\right) \cdot p_{p}
$$

Pressure recovery coefficient $c_{p c}$ can be determined in a similar way using relation 2). Calculation of mass flow through the centric orifice $\dot{m}_{\text {orifice }}$ uses the corresponding standard ČSN EN ISO 5167-2 [6]. The calculation itself was implemented using the Matlab software. No device for mass flow measurement is placed on the wind tunnel. For this reason the mass flow $\dot{m}_{\text {tunnel }}$ was defined from the continuity equation using the relation:

$$
\dot{m}_{\text {tunnel }}=\rho_{1} \cdot w_{1 a x} \cdot S_{1} .
$$

Measurement was done only for Var. 6. First the air was blown in an empty exhaust hood (without any struts and supports). This variant was labelled as the Reference Variant. Then the hood was supplemented with flat struts the so called Var. e. Last time the 
measurement was done with circular struts - Var. $f$. The hood supplemented with flat struts for measurement without blowing showed slightly better results in comparison with circular struts. The values of pressure recovery coefficient will be given later in the paper. The placement of individual struts and individual measured variants are shown in Fig. 4. Measurements were done for the same values of Mach number as in previous experiments, namely $0.2 \mathrm{Ma}$ and $0.4 \mathrm{Ma}$, so that the results obtained could be compared.

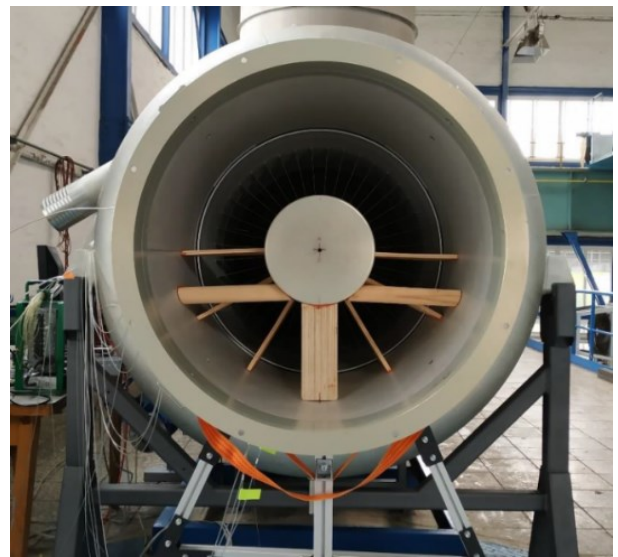

a)

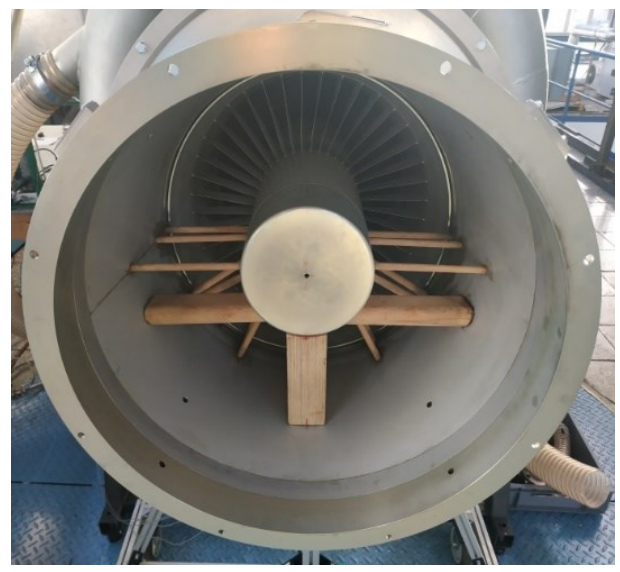

b)

Fig. 4. a) Flat struts - Var. $e$; b) Circular struts - Var. $f$.

During the experiment the maximum mass flow of the blown air is about $0.6 \mathrm{~kg} \cdot \mathrm{s}^{-1}$, which is given by dimensioning of the whole air system. For $0.2 \mathrm{Ma}$ it is about $8.5 \%$ of the mass flow through the wind tunnel and for $0.4 \mathrm{Ma}$ it is about $4.5 \%$. The mass flow of the blowing pipe is measured using the centric orifice, which can be seen in Fig. 5 a). Behind the centric orifice there is a limiting orifice used for adjusting the blowing pressure to $1 \mathrm{bar}$. From the blowing track the air goes through one stop valve that enables regulation of the air flow to the torus. From the torus the air is driven around the perimeter into the blowing slot, see Fig. 5 b). The main air flow is marked in blue, the blown air in red and the joined air in green.

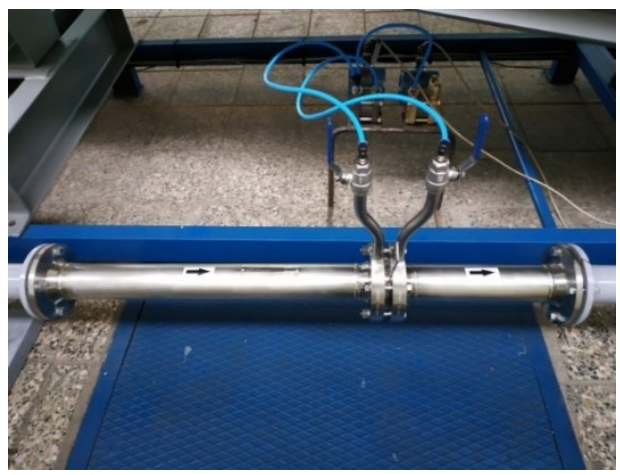

a)

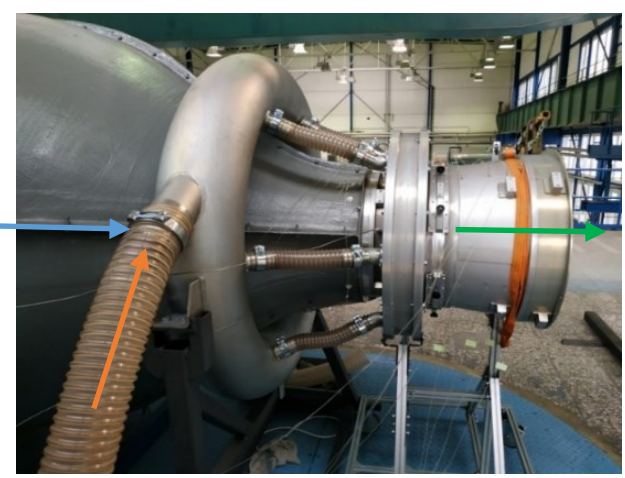

b)

Fig. 5. a) Centric orifice plate; b) Blowing into axial exhaust hood.

The course of the pressure recovery coefficient of the exhaust hood supplemented with a circular extension pipe in dependence on the ratio of mass flow blown through the slot and the mass flow through the wind tunnel for an empty hood (Var. 6) can be seen in Figure 6. 
From the picture it is evident that for both values of Mach number there is a decrease in value $c_{p}$. Minimal value is in both cases reached for blowing $2 \%$ of mass flow through the wind tunnel. Later there is an increase in value $c_{p}$. For lower value of Mach number the pressure coefficient values increase steeply. The positive influence of blowing is manifested from about $6.5 \%$. The highest value of $c_{p}$, i.e. $c_{p}=0.694$ is reached for blowing about $8.5 \%$ of the mass flow through the wind tunnel. The increase is about 2 percentage points. Due to blowing track dimensions it was not possible to further increase the amount of blown air. For this reason it was not possible to find the value of the blown amount in which $c_{p}$ does not grow any more. For higher values of Mach number blowing does not have an entirely positive influence. However, it can be assumed that the course would be similar to that in the first case and the positive effect of blowing would be minimal. The real blown amount is about at the level of $2 \div 2.5 \%$ from the flow through the tunnel.

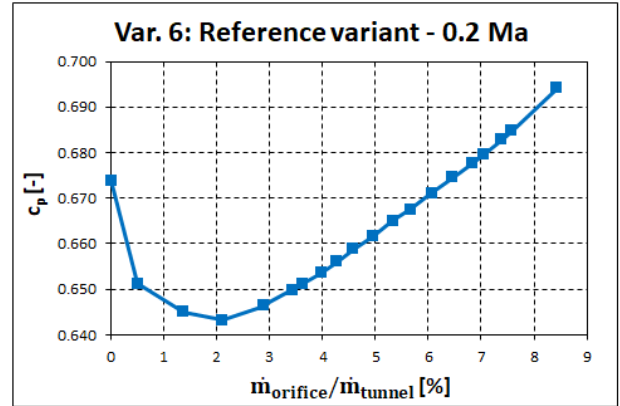

a)

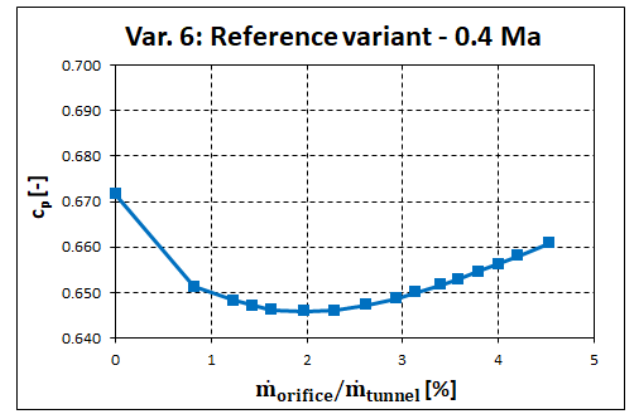

b)

Fig. 6. a) Blowing - Reference variant 0.2 Ma; b) Blowing - Reference variant 0.4 Ma.

In Figure 7 it is possible to observe three courses of $c_{p}$ in dependence on length $L$ of the exhaust hood. The case without blowing is marked in blue, the course with $2 \%$ is in green and the course with blowing maximal amount of air considering the track dimensions is in red. The end of the exhaust hood is marked with a black vertical line. For lower Mach number values it is evident that when blowing a small amount of air, the pressure coefficient is lower in all assessed planes. On the other hand when blowing a maximal amount the pressure coefficient reaches higher values in all assessed planes. For a higher value of Mach number it can be observed that the pressure coefficient is in all assessed planes lower than in the case without blowing.

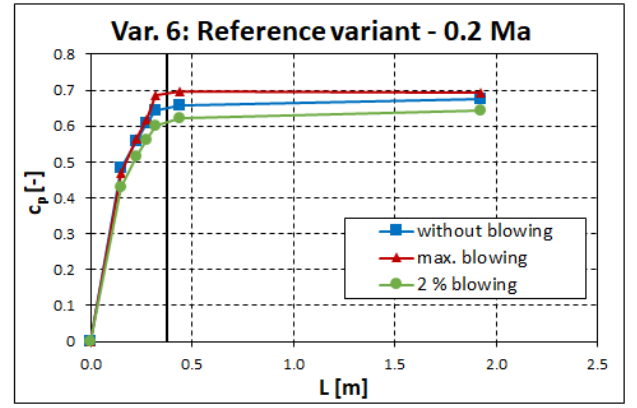

a)

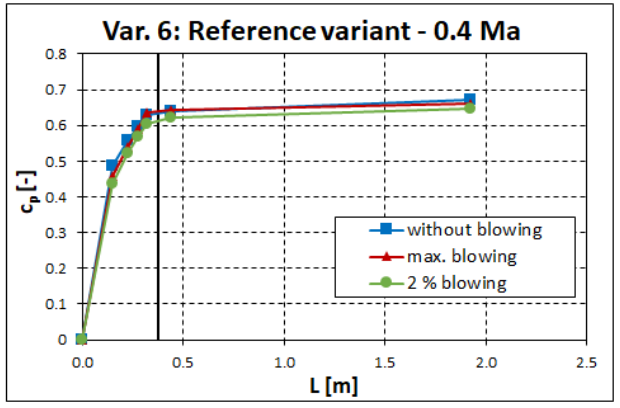

b)

Fig. 7. Dependence $c_{p}$ on $L$ : a) Blowing - Ref. variant $0.2 \mathrm{Ma}$; b) Blowing - Ref. variant $0.2 \mathrm{Ma}$. 
The course of pressure recovery coefficient in dependence on the ratio of mass flow blown through the slot and the mass flow through the wind tunnel in the case when the exhaust hood is supplemented with flat struts (Var. $e$ ) can be seen in Figure 8. Similarly to the previous case, even here first the pressure coefficient value decreases for both level of Mach number. Minimal value $c_{p}$ is reached again for blowing about $2 \%$ of mass flow through the wind tunnel. The positive influence of blowing compared to the variant without blowing appears approximately from the value $4.2 \%$ for both levels of Mach number. Unlike the reference variant it is possible to observe a positive influence of blowing even for higher values of Mach number. However, the increase of the value is not significant. For the higher value of Mach number the course is practically constant. Not even in this case the point (extreme) was found to show at what value the pressure coefficient will again decrease.

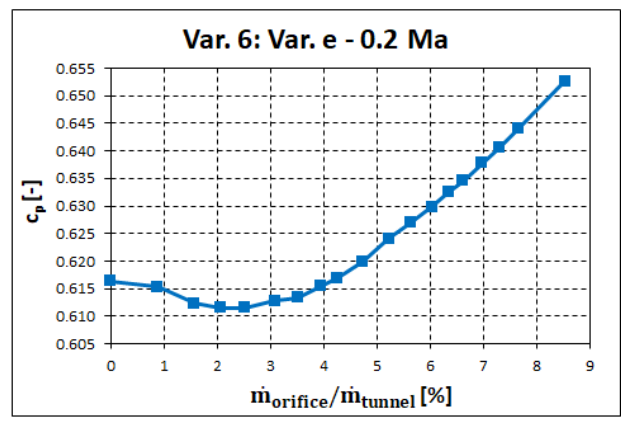

a)

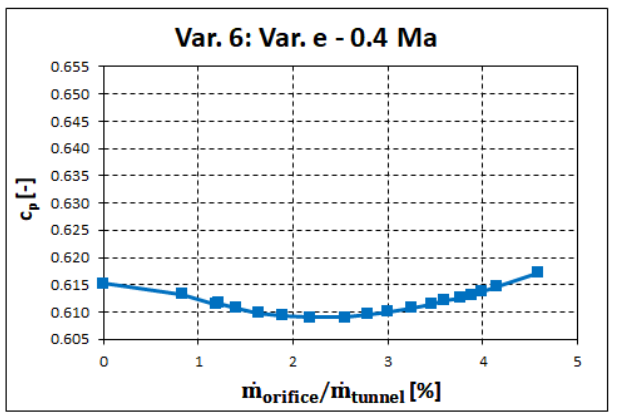

b)

Fig. 8. a) Blowing - Var. e 0.2 Ma; b) Blowing - Var. e 0.4 Ma.

To illustrate, the dependence of pressure coefficient $c_{p}$ on length $L$ of the exhaust hood supplemented with a circular extension pipe was plotted again, see Figure 9. The colour coding is similar to the reference variant. Here the black vertical lines mark the beginning and ending of the inner struts and supports. In the hood supplemented with inner struts the increasing tendency of static pressure is significantly slowed down in comparison to the reference variant, which is caused by the presence of struts. The supports reduce the diffuser cross section, which is demonstrated by local increase of flow velocity and by increase of local losses during the passage of the medium. The static pressure is restored and increases steeply in the remaining part of the diffusor. The pressure coefficient in the circular pipe is growing more moderately, which is given by only gradual stabilization of the flow and complete filling of the flow section.

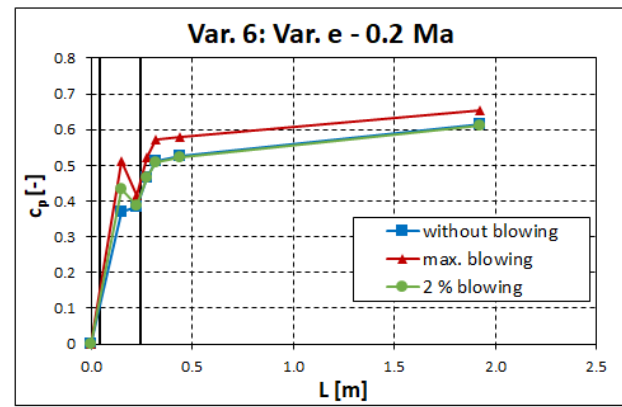

a)

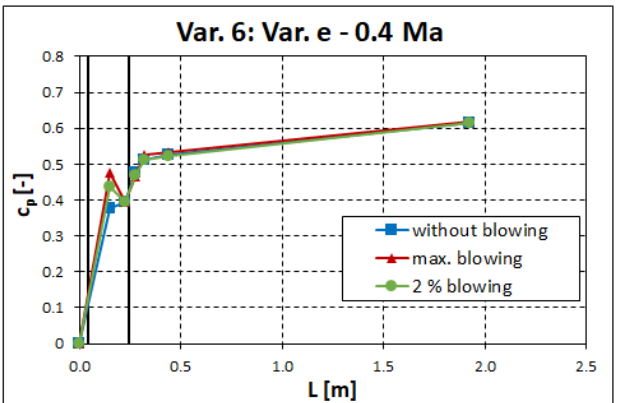

b)

Fig. 9. Dependence $c_{p}$ on $L$ : a) Blowing - Var. e 0.2 Ma; b) Blowing - Var. e 0.4 Ma. 
For a lower value of Mach number it can be observed that during the blowing the value $c_{p}$ assessed in the first plane grows rather significantly. In the following plane the values are approximately the same. During maximal blowing the pressure coefficient reaches higher value in all assessed planes in comparison to the variant without blowing. For $2 \%$ blowing the values are approximately the same in all assessed planes. For a higher value of Mach number the influence of blowing is minimal. It is only demonstrated by an increase of pressure coefficient value assessed to the first plane of the exhaust hood. In the following planes the course is almost identical with the case when no air was blown to the slot.

As the last one the variant of blowing with round struts was measured (Var. $f$ ), see Figure 10. Again it can be observed that the lowest levels of $c_{p}$ are reached approximately during $2 \%$ blowing. The courses are very similar to the flat struts variants. The positive influence of blowing compared to the variant without blowing is evident from the value of $4.5 \%$ for both values of Mach number.

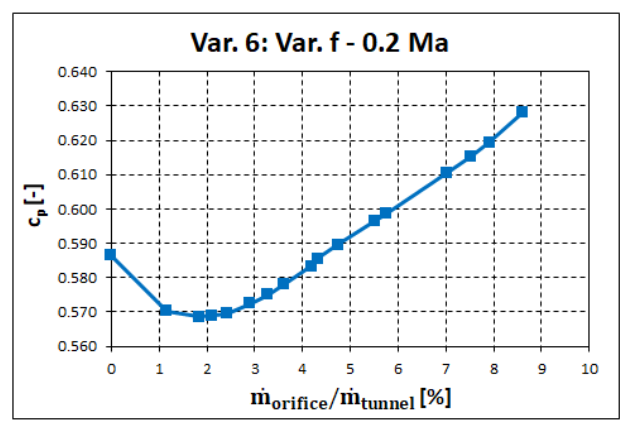

a)

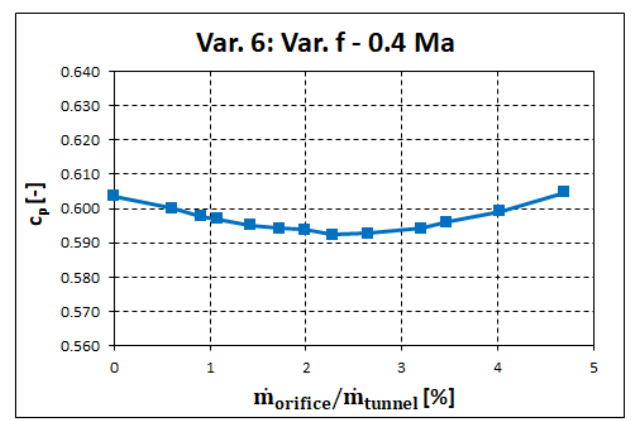

b)

Fig. 10. a) Blowing - Var. $f 0.2 \mathrm{Ma}$; b) Blowing - Var. $f$ 0.4 Ma.

To illustrate, the dependence of pressure coefficient $c_{p}$ on the exhaust hood length $L$ supplemented with a circular extension piped was plotted in the graph, see Figure 11. Colour marking is the same as in previous examples. Black vertical lines mark the beginning and ending of inner struts. For a lower level of Mach number it can be observed that during blowing the $c_{p}$ value, related to the first plane, increases again. This increase is not as significant as it was for variants with flat struts. In the case of $2 \%$ blowing the values are lower in all assessed planes in comparison with the variant without blowing. For higher values of Mach number the influence of blowing is again minimal.

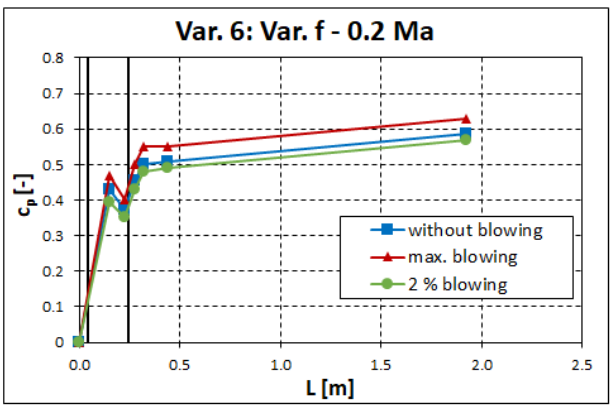

a)

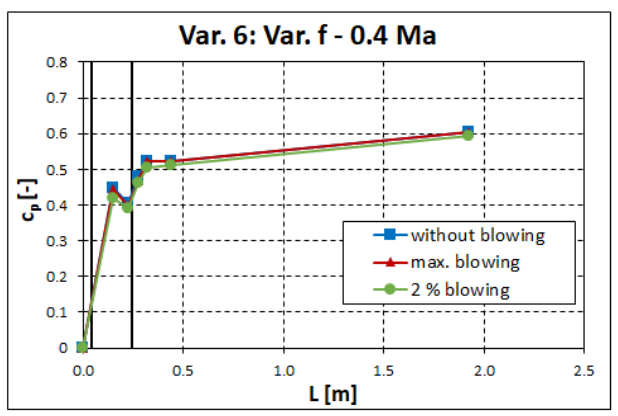

b)

Fig. 11. Dependence $c_{p}$ on $L$ : a) Blowing - Var. $f 0.2 \mathrm{Ma}$; b) Blowing - Var. $f$ 0.4 Ma. 
From the results obtained it is evident that a more significant improvement of the pressure coefficient only occurs for lower value of Mach number, or for higher blown volume. The highest value is in all cases reached for maximal blown volume of air (with the exception of the reference variant $-0.4 \mathrm{Ma}$ ). In Table 1 it is possible to observe the values of pressure coefficient for the variant without blowing, with maximal blown amount and the increase (or decrease) of $c_{p}$.

Table 1. Blowing into axial exhaust hood.

\begin{tabular}{|c|c|c|c|c|c|c|}
\hline \multirow{2}{*}{ Var. 6 } & \multicolumn{2}{|c|}{ Reference var. } & \multicolumn{2}{c|}{ Var. $\boldsymbol{c}$} & \multicolumn{2}{c|}{ Var. $\boldsymbol{f}$} \\
\cline { 2 - 7 } & $0.2 M a$ & $0.4 M a$ & $0.2 M a$ & $0.4 M a$ & $0.2 M a$ & $0.4 M a$ \\
\hline$c_{p}$ - without blowing [-] & 0.674 & 0.672 & 0.616 & 0.615 & 0.586 & 0.604 \\
\hline$c_{p}$ - max. blowing [-] & 0.694 & 0.661 & 0.653 & 0.619 & 0.628 & 0.605 \\
\hline+ increase (- decrease) $c_{p}[-]$ & $\mathbf{0 . 0 2}$ & $\mathbf{- 0 . 0 1 1}$ & $\mathbf{0 . 0 3 6}$ & $\mathbf{0 . 0 0 4}$ & $\mathbf{0 . 0 4 2}$ & $\mathbf{0 . 0 0 1}$ \\
\hline
\end{tabular}

In Figure 12 it is possible to observe the dependence of pressure recovery coefficient ratio $c_{p} / c_{p 0}$ on the velocity ratio $c_{\text {blowing }} / c_{\text {main }}$ for Var. $e$, where:

$c_{p} \quad-$ pressure coefficient with air flow blowing,

$c_{p 0} \quad-$ pressure coefficient without air flow blowing,

$c_{\text {blowing }}-$ velocity of blown air,

$c_{\text {main }} \quad-$ velocity of main flow through wind tunnel.

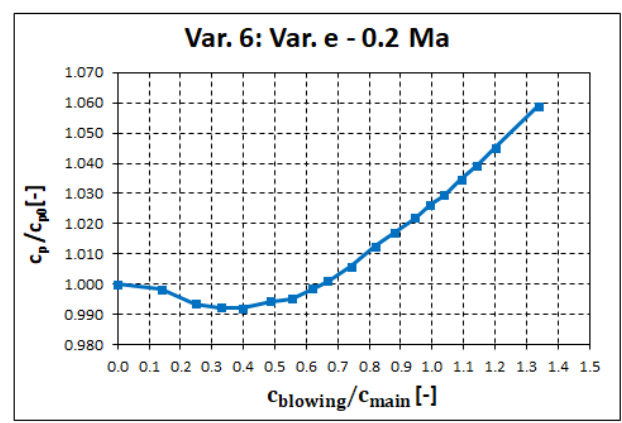

a)

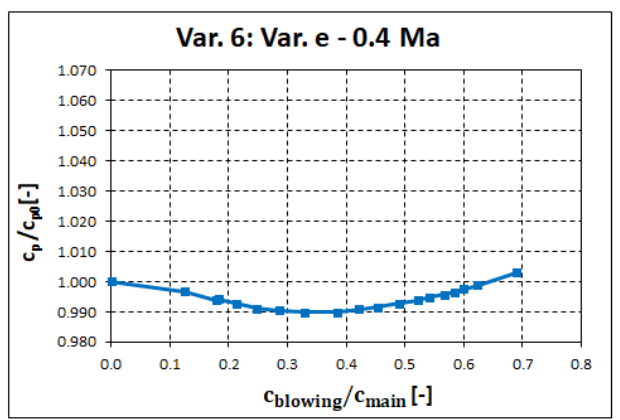

b)

Fig. 12. Dependence $c_{p} / c_{p 0}$ on $c_{\text {blowing }} / c_{\text {main }}$ : a) Var. e 0.2 Ma; b) Var. e 0.4 Ma.

Based on graphs it is possible to observe certain similarity with Fig. 11 . The minimal value of the pressure recovery coefficient ratio is within the velocity ratio range. $(0.3 ; 0.4)$. The increase of the pressure recovery ratio occurs in all cases. Due to blowing track dimensions it was not possible to further increase the amount of blown air. For this reason it was not possible to find the second extreme and to prove the similarity of both experiments in their entirety. However, it should be noted that blowing intensity higher than about $2.5 \%$ is outside the real range of steam turbine operation. 


\section{Conclusion}

In practice it is not desirable to take a big amount of steam from hollow rotor blades and thus also to blow this steam into the slot. In axial exhaust hoods blowing is only used for returning steam to the cycle. Normally the amount ranges from 2 to $2.5 \%$ with respect to the operating mode. From the obtained results it is evident that while blowing $2 \%$ is least suitable, for values higher than $4 \%$ the positive influence begins to manifest. In general it can be said that it is more suitable to blow a higher amount of steam. However, this steam would not be used for work in the last stage, which as a result would mean much bigger losses at the whole turbomachine output. Even in the case of blowing $2 \%$ the decline in $c_{p}$ is not essential. In general, blowing does not have much benefit for axial exhaust hoods, it is only a safe and structurally simple way to return the sucked steam back to the system.

\section{References}

1. M. Hoznedl, Difuzorová prouděni se specifickými okrajovými podmínkami. Plzeň, (2007)

2. Volkmer, S.*, Hirschmann, A.*, Casey, M.*, Montgomery, M.**. The impact of a leakage jet on flow separation in axial gas turbine diffusers. *ITSM, Institute of Thermal Turbomachinery, Universität Stuttgart, Germany, **Siemens AG, Gas turbine, Florida, USA

3. R. Kalista, L. Mrózek, V. Sláma, M. Vrátný, K. Sedlák, B. Rudas, M. Tmej, Experimental measurement on the air test rig of the axial steam turbine exhaust casing. AIP Conference Proceedings 2047, 020006 (2018), DOI: 10.1063/1.5081639

4. R. Kalista, L. Mrózek, V. Sláma, K. Sedlák, Steam Turbine Axial Exhaust Diffuser Investigation Using a Test Rig and its Numerical Model. Proceedings of the ASME 2019 International Mechanical Engineering Congress and Exposition. Volume 6: Energy. Salt Lake City, Utah, USA. November 11-14, (2019) V006T06A033. ASME. https://doi.org/10.1115/IMECE2019-10267

5. D. Tupý, V. Sláma, R. Kalista, Experimental measurement on the air test rig of the axial steam turbine exhaust casing, (2019) [in press]

6. Evropská Norma. Měrení průtoku tekutin pomocí snímaču diferenčního tlaku vložených do zcela zaplnéného potrubí kruhového prưrezu - Část 2: Clony. ČSN EN ISO 5167-2. (2003) 